\title{
Personalien
}

\section{Jürgen Krause †}

DOI 10.1515/iwp-2016-0041

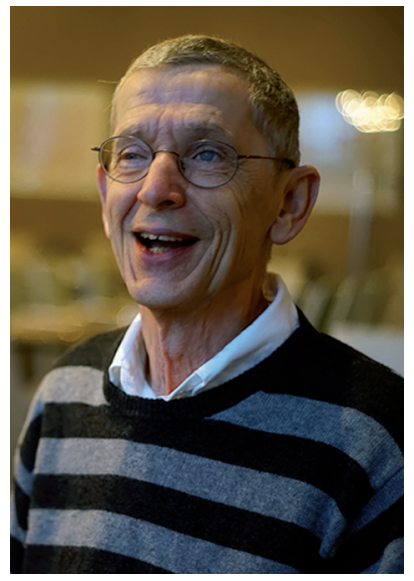

Jürgen Krause im Februar 2014 in Berlin (nach der Verabschiedung von Michael Kluck bei der SWP), Foto: Wolff
Prof. Dr. Jürgen Krause, geboren am 21. Februar 1944 in Liebenthal (Schlesien), ist am 6. März $2016 \mathrm{im}$ Alter von 72 Jahren plötzlich und unerwartet verstorben.

Seine akademische Laufbahn begann 1971 an der Universität Regensburg, wo er nach dem Studium der Germanistik, Geschichte und Sozialkunde in Würzburg und Regensburg als wissenschaftlicher Angestellter für Nichtnumerische Datenverarbeitung arbeitete. Die Promotion in Deutscher Philologie und Allgemeiner Sprachwissenschaft erfolgte im Jahr 1976 mit einer Dissertation zu den Verben des Geschehens. 1978 ging er als externer Fellow an das Wissenschaftliche Zentrum der IBM in Heidelberg und kehrte ein Jahr später an das neue Fachgebiet der Linguistischen Informationswissenschaft der Universität Regensburg zurück, wo er 1981 habilitierte. Die Habilitationsschrift trug den Titel „Die natürliche Sprache als Abfragesprache von Fakten-Informationssystemen" und wurde unter dem Titel „Mensch-Maschine-Interaktion in natürlicher Sprache“ in der Reihe Sprache und Information im Niemeyer Verlag veröffentlicht. Bald darauf erhielt Jürgen Krause einen Ruf auf die C3-Professur für Linguistische Informationswissenschaft an der Universität Regensburg, die er bis ins Jahr 1994 innehatte. In etwa zeitgleich übernahm er den Vorsitz der Gesellschaft für Linguistische Datenverarbeitung (GLDV), heute GSCL, und stand dem Verband bis 1993 als Beiratsmitglied und (zusammen mit Peter Hellwig) als Herausgeber der Buchreihe Sprache und Computer zur Verfügung. An der Universität Regensburg baute er die (Linguistische) Informationswissenschaft als Lehr- und Forschungsgebiet auf und begründete den gleichnamigen Studiengang. Sein Forschungsinteresse galt der automatischen Indexierung, dem Information Retrieval, der benutzungsfreundlichen Mensch-Maschine-Interaktion und Softwareegonomie, wobei er stets eine empirisch-bewertende Perspektive einnahm. Diese Themenfelder ziehen sich durch seinen gesamten Werdegang, verschieben sich aber hin zu aktuellen Varianten (z. B. der Computervisualistik).

Viele große, durch Drittmittel finanzierte Projekte fallen in die Regensburger Zeit: Das vom damaligen Bundesministerium für Forschung und Technologie geförderte Projekt PADOK beschäftigte sich mit dem Aufbau einer automatischen Erschließung von Patentdokumenten für die Deutsche Patentdatenbank PATDPA. Partner waren das Deutsche Patentamt, das Fachinformationszentrum Karlsruhe, die GID/GMD und mehrere Patentabteilungen großer Unternehmen. Später kam als umfangreiches, vom Bundeswirtschaftsministerium gefördertes Projekt WINGIIR hinzu, in dem es um den benutzungsfreundlichen $\mathrm{Zu}$ griff auf Werkstoffdatenbanken durch Integration Intelligenter Information-Retrieval-Verfahren ging. 1986 nahm Jürgen Krause einen weiteren Forschungsaufenthalt am Wissenschaftlichen Zentrum der IBM in Heidelberg in der Gruppe Expertensysteme sowie eine Gastprofessur an der Universität Salzburg wahr. Neben seiner intensiven Forschungs-, Publikations- und Herausgebertätigkeit beteiligte sich Jürgen Krause in der Selbstverwaltung der Universität Regensburg - von 1993 bis 1995 als Dekan der Philosophischen Fakultät IV. Er war Gründungsmitglied des Hochschulverbands Informationswissenschaft, zu dessen Vorsitzenden man ihn im Jahr 1994 wählte; dieses Amt hatte er vier Jahre inne. 1996 richtete er in Berlin das Internationale Symposium für Informationswissenschaft (ISI) aus. Im Jahr 2000 gehörte er zu den Initiatoren der Tagungsreihe „Mensch \&amp; Computer“, heute eine der größten Informatiktagungen im deutschsprachigen Raum.

Anfang 1995 ging Jürgen Krause als Wissenschaftlicher Direktor an das Informationszentrum Sozialwissenschaften (IZ) nach Bonn. Dieser Wechsel war mit einem Ruf auf die C4-Professur für Informatik (Schwerpunkt Softwareergonomie und informationelle Prozesse) der Universität Koblenz-Landau verbunden. Viele neue Aufgaben kamen hinzu, z. B. als Vertreter der Deutschen Gesellschaft für Soziologie (DGS) in der IuK-Kommission der wissenschaftlichen Fachgesellschaften in Deutschland und in der Kommission Informationsgesellschaft im Deutschen Institut für Normung (DIN). An der Universität Koblenz beteiligte er sich maßgeblich am Aufbau des Studiengangs Computervisualistik.

Nach der Evaluation der Gesellschaft Sozialwissenschaftlicher Infrastruktureinrichtungen (GESIS) durch den 
Wissenschaftsrat im April 1997 wurde Jürgen Krause Sprecher des Vorstands und ab März 1999 Geschäftsführender Direktor von GESIS.

Seine Forschungstätigkeit knüpfte an die Regensburger Zeit an und fokussierte auf die Optimierung von Information-Retrieval-Systemen durch die Verbesserung der Interaktion einerseits, und den Ausbau der technischen Leistungsfähigkeit der Systeme andererseits. Systematisiert wurde seine Forschung in zwei von ihm formulierten Modellen: Im WOB-Modell (auf der Werkzeugmetapher basierende strikt objektorientierte graphisch-direktmanipulative Benutzungsoberflächen) bündelt er Vorschläge, um die bei der Gestaltung von Benutzungsoberflächen inhärenten Widersprüche der Zielsetzung auszugleichen. Im Schalen-Modell formulierte er erstmals 1996 nicht nur eine Strategie zur technischen Vernetzung verschiedener, und vor allem unterschiedlich tief erschlossener Informationsquellen, sondern übergeordnet auch eine Strategie für das Zusammenspiel von klassischer Fachinformation und den durch das Internet neu erreichbaren zahlreichen alternativen Informationsanbietern. Der Titel seiner programmatischen Schrift „Informationserschließung und -bereitstellung zwischen Deregulation, Kommerzialisierung und weltweiter Vernetzung " zeigt deutlich die Zeitenwende, der sich die Fachinformation in Krauses Jahren bei GESIS stellen musste. Dabei gelang es ihm, die Fachinformation aus der Rechtfertigungsrolle in eine Führungsrolle zu bringen.

Krause war Mitglied in den Beiräten des Deutschen Instituts für Internationale Pädagogische Forschung (DIPF), des Instituts für Wirtschaftsforschung (HWWA), der Stiftung Wissenschaft und Politik (SWP), des Bundesinstituts für Sportwissenschaft (BISp), um nur einige zu nennen. Für die Deutsche Forschungsgemeinschaft (DFG) fungierte er als Mitglied des Bibliotheksausschusses, des Unterausschusses für Datenverarbeitung und Kommunikationstechniken sowie für Informationsmanagement. Neben seinen vielfältigen Gutachter- und Beratungstätigkeiten war Krause ab August 2003 Sprecher des Steuerkreises Informationsverbund Pädagogik - Sozialwissenschaft Psychologie (infoconnex) und Mitglied der Lenkungsgruppe bzw. später des Vorstands des Deutschen Wissenschaftsportals vascoda.

Im April 2009 ging die berufliche Laufbahn von Prof. Dr. Jürgen Krause zu Ende und er ließ sich mit seiner Frau Ingrid in Schwerin nieder. Sieben Jahre konnten beide dort den gemeinsamen Ruhestand genießen.

Auf die Todesnachricht kamen von vielen Kollegen und Kolleginnen, ehemaligen Mitarbeitern und Mitarbeite- rinnen, von Regensburger und Koblenzer Absolventinnen und Absolventen und aus dem Kreis der ehemaligen Doktorandinnen und Doktoranden viele Rückmeldungen, die die hohe Wertschätzung für Jürgen Krause und den Dank für sein Engagement für jeden Einzelnen ausdrückten. Die Fachwelt verliert mit ihm einen hochgeschätzten Kollegen und geradlinigen, kritischen Denker und Wissenschaftler, der vorgelebt hat, sich auch außerhalb ausgetretener Pfade zu bewegen. Jürgen Krause verstand es zeitlebens, die Disziplinen der Informationswissenschaft und Informatik zu verbinden.

Wir werden ihn als starken Mentor, als faszinierende Persönlichkeit und warmherzigen Freund in Erinnerung behalten und es wird viele Situationen geben, in denen wir ihn vermissen werden.

Im Namen der Kolleginnen und Kollegen aus Informatik und Informationswissenschaft Maximilian Eibl, Christian Wolff und Christa Womser-Hacker

\section{Reinhard Supper-Jüdefeld $t$}

Am 21. April 2016 verstarb der ehemalige Vorstandsvorsitzende des Berliner Arbeitskreises Information (BAK) Reinhard Supper-Jüdefeld. Mit ihm verliert die DGI den langjährigen verlässlichen Ansprechpartner zu ihrem Berliner Regionalverband. Als Stellvertretender Vorsitzender der Arbeitsgemeinschaft der Spezialbibliotheken wirkte er als unermüdlicher Brückenbauer zwischen den Welten von Bibliothek und Information. In Vertriebsgesprächen und vor Ort bei Besuchen sowie Präsentationen bleibt der langjährige Leiter der Bibliothek der Bundesanstalt für Materialforschung und -prüfung (BAM) in Berlin als ein Mensch der leisen Töne, des Zuhörens und einer hohen Empathiefähigkeit in Erinnerung. Nur folgerichtig, dass er bereits vor seinem Wechsel in die Nachberufsphase diese Gabe, sich in andere hineinzuversetzen, in die Ausbildung und eine Praxis für Psychotherapie investiert hat.

Mit Reinhard Supper verliert die Informationswissenschaft- und -praxis einen außergewöhnlichen Menschen. Unsere Anteilnahme gilt seinen Angehörigen und Weggefährten.

Dr. Luzian Weisel im Namen des Vorstandes der DGI 